\title{
A Quaternion-based Approach to Estimate Respiratory Rate from the Vectorcardiogram
}

\author{
Daniel Romero ${ }^{1}$, Jesús Lázaro ${ }^{2,3}$, Raimon Jané ${ }^{1,3,4}$, Pablo Laguna ${ }^{2,3}$, Raquel Bailón ${ }^{2,3}$ \\ ${ }^{1}$ Institute for Bioengineering of Catalonia (IBEC-BIST), Barcelona, Spain \\ ${ }^{2}$ Aragon Institute for Engineering Research (I3A), University of Zaragoza, Zaragoza, Spain \\ ${ }^{3}$ Biomedical Research Networking Center of Bioengineering, Biomaterials and Nanomedicine, Spain \\ ${ }^{4}$ Universitat Politècnica de Catalunya, BarcelonaTech, Barcelona, Spain
}

\begin{abstract}
A novel ECG-derived respiration (EDR) approach is presented to efficiently estimate the respiratory rate. It combines spatial rotations and magnitude variations of the heart's electrical vector due to respiration. Orthogonal leads $X, Y$ and $Z$ from 10 volunteers were analyzed during a tilt table test. The largest vector magnitude (VM) within each QRS loop was assessed, and its $3 D$ coordinates were converted into unit quaternion $\boldsymbol{q}_{\boldsymbol{b}}$. Angular distances between these quaternions and the axes of the reference coordinate system, $\theta_{x}, \theta_{y}$ and $\theta_{z}$, were then computed as EDR signals to track their relative variations caused by respiration. The respiratory rate was estimated on the spectrum of individual EDR signals obtained from the angular distances and VM time-series, but also on EDR signals obtained by principal component analysis (PCA). Relative errors $\left(e_{R}\right)$ to the reference respiratory signal exhibited relatively low values. The combination of EDR signals' spectrum $\left\{\theta_{X}, \theta_{Y}, \theta_{Z}, V M\right\}\left(e_{R}=0.63 \pm 4.15 \%\right)$ and individual signals derived from $\theta_{X}\left(e_{R}=0.46 \pm 8.22 \%\right)$ and PCA $\left(e_{R}=0.36 \pm 6.58 \%\right)$ achieved the overall best results. The proposed method represents a computationally efficient alternative to other EDR approaches, but its robustness should be further investigated. The method could be enhanced if combined with other features tracking morphological changes induced by respiration.
\end{abstract}

\section{Introduction}

Measuring respiratory rate represents an important task in many clinical applications, especially in those involving patients with chronic respiratory disorders. Different techniques are used in clinical practice for this purpose, such as plethysmography or pneumography, based on impedance measurements. Other techniques use airflow measures including volumen (spirometry), temperature (thermistors) or pressure (pressure transducer). Despite their accuracy, the above techniques might be uncomfortable and invasive, and are not suitable for continuous, long-term monitoring in the clinical setting, at home or in sport applications .

In particular, real-time respiration monitoring with noninvasive devices can be useful for studying exercise physiology, patients' sleep and other dynamic conditions [1]. However, obtaining accurate and robust respiratory information during such scenarios might be challenging. A potential solution is clearly sighted from the proliferation of wearable devices, many of them provided with several physiological sensors. The wide range of indirect methods proposed in the literature to extract respiratory information make it much more suitable. Among these methods, the electrocardiogram (ECG)-derived respiration (EDR) signals are commonly used with either one or several ECG channels [2]. Since respiratory information affects both the ECG rhythm and morphology, a convenient method would be one that combines morphological information from all leads, as occurs with respiration-induced rotations of the heart's electrical vector.

In this study, we aimed at implementing an efficient EDR approach based on quaternions to estimate respiratory rate. The approach combines the spatial rotations and magnitude variations of the heart's electrical vector from the vectorcardiogram (VCG). Using quaternions provides us with a compact mathematical notation for 3D orientations and rotations, leading to more efficient rotation operations compared to Euler's angles or rotation matrices.

\section{Material and Methods}

\subsection{Tilt test database}

The database comprises 10 healthy volunteers subjected to a tilt table test, aged $28.5 \pm 2.5$ years. The protocol included an initial basal period of $4 \mathrm{~min}$ in supine position, followed by $5 \mathrm{~min}$ tilted head-up to $70^{\circ}$, and a final 4 min period in supine position. Transition periods between stages lasted $18 \mathrm{~s}$. For all subjects, standard 
12-lead ECG recordings were acquired at $1000 \mathrm{~Hz}$ using Biopac ECG100C. The respiratory signal was recorded using RSP100C amplifier and TSD201 transducer sampled at $125 \mathrm{~Hz}$. More details on this database can be found in [3].

\subsection{ECG Preprocessing}

ECG signals were preprocessed before the analysis and estimation of respiratory rate, including automatic QRS complex detection and delineation [4] and visual inspection to remove abnormal beats, baseline drift attenuation, $4^{\text {th }}$ order bidirectional Butterworth low-pass filtering at 45 $\mathrm{Hz}$ to remove power line interference and high frequency noise. These steps were applied to orthogonal leads $X, Y$ and $Z$, derived from standard leads (V1-V6, I and II) using the Dower inverse matrix. The maximum vector magnitude within each QRS complex loop, $\mathrm{VM}_{\text {max }_{i}}$, was determined using a 140-ms segments starting $10 \mathrm{~ms}$ before the QRS onset points. The 3D points coordinates $x_{i}, y_{i}$ and $z_{i}$, associated with the $\mathrm{VM}_{\max _{i}}$ of the $i$-th beat, were used in further analysis to computed the proposed measures.

\subsection{Orientation and rotation quaternions}

To estimate the respiratory rate from the ECG, we exploit the advantages of an efficient approach to represent and compute 3D spatial rotations based on quaternions [5]. Quaternions are basically a numerical compact notation for 3D rotation and orientations, in the form of:

$$
\boldsymbol{q}=\left[q_{0}, q_{1}, q_{2}, q_{3}\right]
$$

where $q_{0}$ is referred to as the real component, and the remaining terms $\left(q_{1}, q_{2}, q_{3}\right)$ are the imaginary components of three mutually orthogonal imaginary unit vectors, $\boldsymbol{i}, \boldsymbol{j}$ and $\boldsymbol{k}$, respectively, which are implied in (1). Thus, quaternions can also be expressed in their extended notation as:

$$
\boldsymbol{q}=q_{0}+\boldsymbol{i} q_{1}+\boldsymbol{j} q_{2}+\boldsymbol{k} q_{3}
$$

Rotation quaternions belong to a subset of quaternions closely related to the axis-angle representation of rotations. According to Euler's rotation theorem, two parameters are needed to specify any 3D rotation: a unit vector $\overrightarrow{\boldsymbol{u}}=[x, y$, $z$ ] that defines an axis of rotation; and an angle $\theta$ representing the magnitude of the rotation about that axis. Thus, an axis-angle rotation can be represented by four numbers as:

$$
[\theta, x, y, z]
$$

and the axis-angle components $[\theta, x, y, z]$ of any rotation can be converted into a rotation quaternion $\boldsymbol{q}$ as follows:

$$
\boldsymbol{q}=\left[\cos (\theta / 2), x \cdot \sin \left(\frac{\theta}{2}\right), y \cdot \sin \left(\frac{\theta}{2}\right), z \cdot \sin \left(\frac{\theta}{2}\right)\right]
$$

From (1) and (4) it can be observed that the real term $q_{0}$ is entirely determined by the rotation angle $\theta$, while the three imaginary terms $\left(q_{1}, q_{2}\right.$ and $\left.q_{3}\right)$ are just the three rotation axis vector scaled by the same factor. A consequence of this representation is that the magnitude of a rotation quaternion is always equal to one.

Quaternions can also be used to rotate a 3D point with respect to the coordinate system, so that the new position of the rotated point can be obtained as follows:

$$
p^{\prime}=q^{-1} p q
$$

where $\boldsymbol{p}$ is the quaternion corresponding to the initial $3 \mathrm{D}$ point $\boldsymbol{p}=[0, x, y, z] ; \boldsymbol{q}$ and $\boldsymbol{q}^{-1}$ represent the rotation quaternion and its conjugate $\left(\boldsymbol{q}^{-1}=\left[q_{0},-q_{1},-q_{2},-q_{3}\right]\right)$, respectively; and $\boldsymbol{p}^{\prime}$ is the quaternion containing the new coordinates position of the rotated 3D point, $\boldsymbol{p}^{\prime}=\left[0, x^{\prime}, y^{\prime}, z^{\prime}\right]$. Note that, for both quaternions $\boldsymbol{p}$ and $\boldsymbol{p}^{\prime}$, the real part $p_{0}$ and $p_{0}^{\prime}$ are always equal to 0 . In these cases, quaternions are called orientation quaternions or attitude quaternions.

\subsection{Angular distances using quaternions}

Now, given two unit quaternions $\boldsymbol{p}$ and $\boldsymbol{p}^{\prime}$ obtained from two spatial 3D points, one can determine the shortest arc representing the angle of rotation from $\boldsymbol{p}$ to $\boldsymbol{p}^{\prime}$. First, we need to find the difference quaternion:

$$
q_{d}=p^{-1} p^{\prime}
$$

Then, the angle of rotation or angular distance $\theta_{d}$ is computed by the following expression:

$$
\theta_{d}=2 \cos ^{-1}\left(\Re\left(\boldsymbol{q}_{\boldsymbol{d}}\right)\right)
$$

where $\Re$ is the real component of $\boldsymbol{q}_{\boldsymbol{d}}$.

Based on Eq. (7), we propose in this work three different measures, denoted as $\theta_{x}, \theta_{y}$, and $\theta_{z}$, evaluating the angular distances between the normalized unit quaternion corresponding to the maximum vector magnitude's coordinates obtained for each QRS loop, and three reference quaternions related to each orthogonal axis, denoted as:

$$
\begin{aligned}
& \boldsymbol{q}_{\boldsymbol{x}}=[0,1,0,0] \\
& \boldsymbol{q}_{\boldsymbol{y}}=[0,0,1,0] \\
& \boldsymbol{q}_{\boldsymbol{z}}=[0,0,0,1]
\end{aligned}
$$

where the normalized unit quaternion related to each $\mathrm{VM}_{\max }$ is obtained by:

$$
\tilde{\boldsymbol{q}}_{i}=\frac{\boldsymbol{q}_{i}}{\left\|\boldsymbol{q}_{i}\right\|}
$$

with $\boldsymbol{q}_{i}=\left[0, x\left(n_{i}\right), y\left(n_{i}\right), z\left(n_{i}\right)\right]$. 


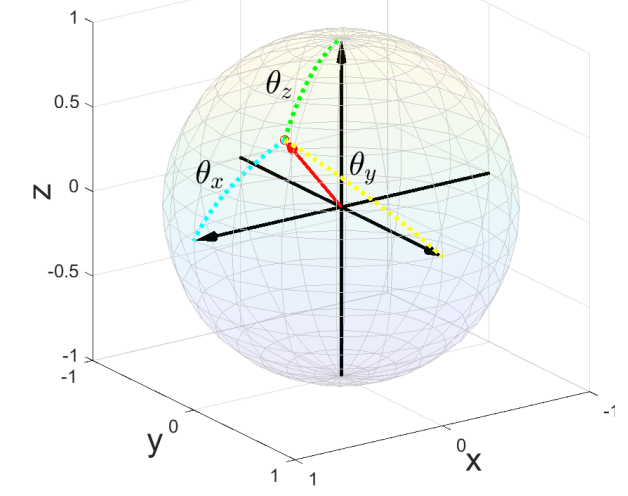

Figure 1. Coordinates of a 3D point associated with the maximum vector magnitude (red arrow) of a real QRS loop, normalized and represented on the unit-ratio hypersphere surface. Arcs in blue, yellow and green represent the angular distance between the 3D point and each axis.

Finally, the values of $\theta_{x}, \theta_{y}$, and $\theta_{z}$ computed over time (see Fig. 1) will be used to generated the EDR signals used for estimating the respiratory rate.

In addition to the angular measures, we evaluated other ECG-derived markers proposed in previous studies to estimate respiratory rate for comparison purpose [2]. These measures represent the upward and downward slopes of the R-wave $\left(s_{U}, s_{D}\right)$, and the shortest angle formed by the intersection of the straight lines resulting from the abovementioned slopes $\left(\angle_{R}\right)$. These markers were evaluated only in the lead called $N$-loop derived lead (NLDL), as it was proposed in [1]. The corresponding EDR signals were thus denoted as $R_{s_{U}}(t), R_{s_{D}}(t)$ and $R_{L_{R}}(t)$. Finally, the time needed for ECG markers computation, was estimated on an Intel Core I7-8750H CPU @ 2.21 GHz, RAM 16 GB.

\subsection{Proposed EDR signals}

Different EDR signals were generated from each marker described in previous section. Four EDR signals were obtained from the angular distances and vector magnitude time series, $\theta_{x}\left(t_{i}\right), \theta_{y}\left(t_{i}\right)$, by $\theta_{z}\left(t_{i}\right)$ and $\mathrm{VM}_{\max }\left(t_{i}\right)$, being $t_{i}$ the time instant associated to the $i$-th beat. An outlier rejection filter based on the median absolute deviation (MAD) was then applied, followed by cubic-splines interpolation at $4 \mathrm{~Hz}$ to get evenly sampled signals. Lastly, a band-pass filter $([0.05,1] \mathrm{Hz})$ was used to retain a wide but conservative frequency range in which the components related to the breathing modulation are expected to be.

In addition to the above signals, we generated a new EDR signal from principal component analysis (PCA), applied to $R_{\theta_{x}}(t), R_{\theta_{y}}(t)$ and $R_{\theta_{z}}(t)$. With this, we obtain a new signal projected onto the first component, defined as $R_{\mathrm{PCA}}(t)$, emphasizing the breathing-related oscillations.

\subsection{Respiratory rate estimation}

Respiratory rate estimation was performed using a robust approach used in [1]. It was applied to individual EDR signals, but also to combinations of signals to obtain more robust respiratory rate estimates. The approach is based on 3 stages, including the estimation of power spectrum (PS), a peak-conditioned average, and the respiratory rate estimation. The PS is obtained from the Welch periodogram, using a running window $k$ of $T_{s}$ duration (42 s) for each signal $l$. An average spectrum, $S_{l, k}(f)$, is obtained every $5 \mathrm{~s}$ from average of PS obtained in subintervals of $12 \mathrm{~s}$ with half overlapping. Then, $H_{s}$ spectra $S_{l, k}(f)$ are peakconditioned average in the second stage. Mathematically, it can be defined as:

$$
\bar{S}_{k}(f)=\sum_{h=0}^{H_{s}-1} \sum_{l} X_{l, k-h}^{A} X_{l, k-h}^{B} S_{l, k-h}(f)
$$

where $X_{l, k-h}^{A}$ and $X_{l, k-h}^{B}$ are two criteria deciding whether $S_{l, k}(f)$ is sufficiently peaked or not. Finally, the respiratory peak is determined as the largest peak inside a reference interval $\Omega_{R}(k)$, updated after each respiratory rate estimate. Further details can be found in [1].

To evaluate the performance of the proposed method, conventional error measures were determined:

$$
\begin{gathered}
e_{\mathrm{A}}(k)=\hat{f}(k)-f_{\mathrm{RES}}(k) \\
e_{\mathrm{R}}(k)=\frac{e_{\mathrm{A}}(k)}{f_{\mathrm{RES}}(k)}
\end{gathered}
$$

where $f_{\mathrm{RES}}(k)$ is the respiratory rate estimated from the reference respiratory signal, $e_{\mathrm{A}}(k)$ and $e_{\mathrm{R}}(k)$ are the absolute and relative errors, respectively, and $\hat{f}(k)$ is the actual estimate of the respiratory rate.

\section{Results}

Figure 2 shows an example of the TF maps derived from the average spectra for the angular measure $\theta_{X}(t)$ and the PCA-derived EDR signal. Respiratory rate estimates from these signals and the reference respiratory signal are also added. The numbers at the top of the panels indicate the errors referred to the reference signal estimates.

Table 1 summarizes the average values (Mean \pm SD) accross the participants of $e_{A}$ and $e_{R}$ obtained for all EDR signals analyzed in this work, including those ones used for comparison purpose. On the other hand, Table 2 displays the average processing time achieved when obtaining the measures that produced the different EDR signals.

\section{Discussion and conclusions}

In this study, we presented a novel approach to estimate respiratory rate from the ECG, based on quaternions. 


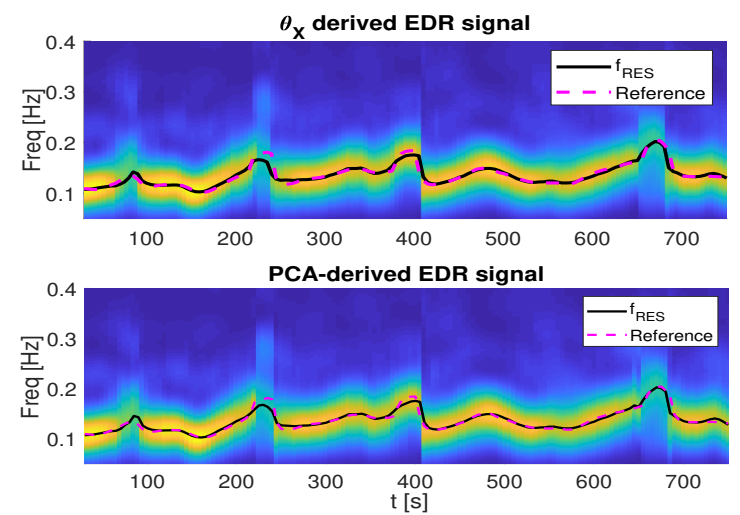

Figure 2. Time-frequency maps obtained from the average spectrum of the EDR signals $R_{\theta_{X}}(t)$ and $R_{P C A}(t)$. Estimates of respiratory rate are plotted for the reference (dashed magente) and the EDR signal (black).

Table 1. Mean $\pm \mathrm{SD}$ of the absolute an relative errors obtained for all patients of the tilt table dataset.

\begin{tabular}{c|cc}
\hline & $e_{A}(\mathrm{mHz})$ & $e_{R}(\%)$ \\
Mean $\pm \mathrm{SD}$ & Mean $\pm \mathrm{SD}$ \\
\hline$R_{\theta_{X}}(t)$ & $-0.06 \pm 1.99$ & $0.46 \pm 8.22$ \\
$R_{\theta_{Y}}(t)$ & $0.04 \pm 2.29$ & $2.08 \pm 10.89$ \\
$R_{\theta_{Z}}(t)$ & $0.22 \pm 1.32$ & $1.64 \pm 7.30$ \\
$R_{\mathrm{PCA}}(t)$ & $-0.04 \pm 1.21$ & $0.36 \pm 6.58$ \\
$R_{\mathrm{VM}}(t)$ & $0.39 \pm 1.21$ & $3.27 \pm 7.98$ \\
$\operatorname{Comb}\left\{R_{\theta_{X}}, R_{\theta_{Y}}, R_{\theta_{Z}}\right\}$ & $-0.06 \pm 1.07$ & $0.68 \pm 5.75$ \\
$\operatorname{Comb}\left\{R_{\theta_{X}}, R_{\theta_{Y}}, R_{\theta_{Z}}, R_{\mathrm{VM}}\right\}$ & $0.04 \pm 0.72$ & $0.63 \pm 4.15$ \\
$R_{\iota_{R}}(t)$ & $-0.26 \pm 1.34$ & $-0.47 \pm 7.85$ \\
$R_{s_{U}}(t)$ & $-0.19 \pm 1.00$ & $-0.56 \pm 5.46$ \\
$R_{s_{D}}(t)$ & $-0.15 \pm 1.19$ & $-0.08 \pm 6.64$ \\
$\operatorname{Comb}\left\{R_{s_{U}}, R_{s_{D}}, R_{\triangle_{R}}\right\}$ & $-0.18 \pm 1.03$ & $-0.50 \pm 5.69$ \\
\hline
\end{tabular}

Table 2. Mean $\pm \mathrm{SD}$ of the mean execution time obtain among all subjects using 10 iterations.

\begin{tabular}{c|c}
\hline ECG-derived features & Elapsed time (ms) \\
Mean $\pm \mathrm{SD}$ \\
\hline$S_{U}+S_{D}$ & $182.0 \pm 25.2$ \\
$S_{U}+S_{D}+\angle_{R}$ & $182.5 \pm 21.3$ \\
$\theta_{x}+\theta_{y}+\theta_{z}$ & $4.7 \pm 8.3$ \\
$\theta_{x}+\theta_{y}+\theta_{z}+\mathrm{VM}_{\max }$ & $45.5 \pm 7.6$ \\
\hline
\end{tabular}

Quaternions provide very high speed for signals processing, stability and robustness to noise arising from numerical inaccuracies caused by floating point computations [5]. We exploited its properties to track the breathing effects on the ECG through the heart's electrical vector rotations.

Our results suggest that the combination of the spectra corresponding to EDR signals derived from the angular distances provides a better performance in relative terms than individual signals. By adding the VM-derived EDR signal spectra to those from the angular measures, and thus incorporating the changes in the magnitude of the cardiac electrical vector, the results became even better and robust. Besides, the error estimates were even lower for the PCAderived EDR signal and $\theta_{X}$, and similar to those of the
QRS slopes and the R angle. However, the proposed angular measures presented a faster execution time, reducing it to more than 30 times in the best case.

The QRS slopes have demonstrated to provide a great performance and robustness, especially in applications using a single ECG channel for the same purpose [2]. However, its computation requires a fitting process that could make it more computationally expensive. Quaternions, by contrast, can be directly generated from the heart electrical vector coordinates, and operating with them involve certain advantages in terms of computational efficiency with respect to Euler's angles and rotation matrices. They are compact, and avoid the problem of "gimbal lock". Further studies are needed to test the robustness of the proposed approach in noisy signals such as those obtained during ambulatory records or sport activities.

\section{Acknowledgments}

This project has received funding from the European Union's Horizon 2020 Research and Innovation Programme under the Marie Sklodowska-Curie grant agreements No. 846636 and No. 745755. Research partly supported by AEI and FEDER under project RTI2018097723-B-I00 and RTI2018 098472-B-I00, by LMP4418 and BSICoS group (T39-20R) funded by Gobierno de Aragón, by the Universities and Research Secretariat (GRC 2017 SGR 01770) and the CERCA Programme from the Generalitat de Catalunya.

\section{References}

[1] Lázaro J, Alcaine A, Romero D, Gil E, Laguna P, Pueyo E, Bailón R. Electrocardiogram derived respiratory rate from QRS slopes and R-wave angle. Annals Biomed Eng 2014; 42(10):2072-2083.

[2] Varon C, Morales J, Lázaro J, Orini M, Deviaene M, Kontaxis S, Testelmans D, Buyse B, Borzée P, Sörnmo L, et al. A comparative study of ecg-derived respiration in ambulatory monitoring using the single-lead ecg. Scientific Reports 2020;10(1):1-14.

[3] Mincholé A, Pueyo E, Rodríguez JF, Zacur E, Doblaré M, Laguna P. Quantification of restitution dispersion from the dynamic changes of the T-wave peak to end, measured at the surface ECG. IEEE Trans Biomed Eng 2010;58(5):1172-82.

[4] Martinez JP, Almeida R, Olmos S, Rocha AP, Laguna P. A wavelet-based ECG delineator: evaluation on standard databases. IEEE Transactions on Biomedical Engineering April 2004;51(4):570-581.

[5] Mukundan R. Quaternions: From classical mechanics to computer graphics, and beyond. In Proc. of the 7th Asian Technology conference in Mathematics. 2002; 97-105.

Address for correspondence:

Daniel Romero

IBEC: Ave. E. Maristany 16, 08019 Barcelona, Spain dromero@ibecbarcelona.eu 